Patenschaftsprogramm zum 94. Deutschen Röntgenkongress

\title{
Die hellsten Köpfe für die Radiologie
}

Medizinstudenten einen eindrucksvollen Einblick in die Vielfalt der Radiologie eröffnen - das ist das Ziel der überaus erfolgreichen Nachwuchsinitiative „die hellsten Köpfe für die Radiologie“. Dank der gemeinschaftlichen Förderung durch individuelle Paten und die Deutsche Röntgengesellschaft konnten in den vergangenen 3 Jahren bereits rund 600 Studenten als „helle Köpfe“ kostenfrei am Deutschen Röntgenkongress teilnehmen.

Wir laden Sie herzlich dazu ein, sich auch 2013 für das Programm zu engagieren und Ihre PJler, Famuli oder Studierenden nach Hamburg zu senden. Dort können die Stipendiaten kostenfrei an allen Veranstaltungen des 94. Deutschen Röntgenkongresses teilnehmen - nicht zuletzt an dem Kongress-Sonderprogramm „Radiologie für Studenten“, das eigens für Studierende angeboten wird. Eine besondere Betreuung und Orientierungshilfe erfahren Kongress-Novizen in der Studentenlounge, die in den vergangenen Jahren hervorragend angenommen wurde. Sie nimmt für den sozialen und fachlichen Austausch einen hohen Stellenwert ein, ebenso wie auch der eigens veranstaltete Hellste-Köpfe-Abend, zu dem die Stipendiaten exklusiv eingeladen sind.

\section{DIE HELLSTEN KÓPFE FÜR DIE RADIOLOGIE}

Machen Sie das Nachwuchsprogramm der Deutschen Röntgengesellschaft auch 2013 wieder zum Erfolg! Übernehmen Sie eine Kongresspatenschaft für Ihren hellsten Kopf und melden Sie ihn ab sofort bei der Deutschen Röntgengesellschaft für das Programm an. Alle Informationen sowie das Anmeldeformular finden Sie unter www.hellste-koepfe.de. Anmeldeschluss ist der 31. Januar 2013.

Für Ihre Rückfragen steht Ihnen die Geschäftsstelle der DRG gerne zur Verfügung. 